\title{
Higher body mass index indicated better overall survival in pancreatic ductal adenocarcinoma patients: a real-world study of 2010 patients
}

Ningzhen Fu ${ }^{1,2,3,4+}$, Yu Jiang ${ }^{1,2,3,4+}$, Kai Qin ${ }^{1,2,3,4}$, Hao Chen ${ }^{1,2,3,4}$, Xiaxing Deng ${ }^{1,2,3,4^{*}}$ and Baiyong Shen ${ }^{1,2,3,4^{*}}$

\begin{abstract}
Background: The association between body mass index (BMI) and the overall survival (OS) of pancreatic ductal adenocarcinoma (PDAC) patients remains controversial and unclear,

Method: A total of 2010 patients from a high-volume center were enrolled in the study. The OS of PDAC patients was evaluated based on restricted cubic spline (RCS), propensity score (PS) and multivariable risk adjustment analyses.

Result: BMI was linearly related to the OS (total $P=0.004$, nonlinear $P=0.124$ ). BMI was analyzed as categorical data based on X-tile software-defined cutoffs and World Health Organization (WHO)-recommended cutoffs. Adjusted with confounding covariates, higher BMI manifested as a positive prognostic predictor. Furthermore, BMI was proven to be associated with the OS in the PS analysis. (Underweight $x_{x t i l e} v$. Normal ${ }_{x t i l e} P=0.003$, Overweight $x_{x t i l e}$ vs. Normal $x_{x t i l e} P=0.019$; Underweight ${ }_{W H O}$ vs. Normal ${ }_{W H O} P<0.001$, Overweight ${ }_{W H O}$ vs. Normal ${ }_{W H O} P=0.024$ ). It was also revealed that patients with higher BMI benefitted more from chemotherapy. (Adjusted hazard ratio (aHR): Underweight $_{\text {xtile }}$ vs. Normal $\left.\right|_{x t i l e}$ vs. Overweight $x_{x t i l e} 0.565$ vs. 0.474 vs. $0.409 ;$ Underweight $_{\text {WHO }}$ vs. Normal WHO $_{\text {vs. }}$ Overweight $_{W H O}: 0.613$ vs. 0.464 vs. 0.425).
\end{abstract}

Conclusion: Among PDAC patients, there was a positive association between BMI and the OS, especially in patients treated with chemotherapy.

Keywords: Body mass index (BMI). Pancreatic ductal adenocarcinoma (PDAC). Overall survival (OS). Restricted cubic spline (RCS). Chemotherapy

\section{Background}

Pancreatic cancer, one of the most lethal malignancies, is estimated to be responsible for 47,050 deaths in 2020 [1]. The 5-year survival rate is only 9\% [1]. Pancreatic ductal adenocarcinoma (PDAC) is the main pattern of

*Correspondence: kejiadxx@hotmail.com; shenby@shsmu.edu.cn

${ }^{\dagger}$ Ningzhen Fu and Yu Jiang are co-first-authors.

${ }^{1}$ Department of General Surgery, Pancreatic Disease Center, Shanghai

Ruijin Hospital affiliated with Shanghai Jiao Tong University School

of Medicine, No.197 Ruijin Er Road, Shanghai 200025, China

Full list of author information is available at the end of the article pancreatic cancer, accounting for approximately $85 \%$ [2]. How to predict and improve the overall survival (OS) of these patients is an long-standing focus of clinicians and researchers.

Body mass index (BMI), an easily accessible and inexpensive parameter, has been reported to be associated with the incidence of pancreatic cancer $[3,4]$. In our previous study, it was discovered that there was an association between higher BMI and better OS among PDAC patients [5]. Thus far, the role of BMI in the long-term prognoses of PDAC patients remains 
controversial [6-10]. Limited by sample size and debatable statistics, in what way BMI affects the OS was not convincingly determined in previous studies. In this study, relying on propensity score (PS) analysis and multivariable risk adjustment analysis, we sought for the association between BMI and the OS. Based on our results, further studies could be designed to explore the underlying mechanisms and relationships between metabolic status and PDAC.

\section{Methods}

Data collection

All eligible patients pathologically diagnosed with PDAC were consecutively enrolled from the Pancreatic Disease Center at the Ruijin Hospital Affiliated to Shanghai Jiao Tong University School of Medicine from 2013.1 to 2019.12. The exclusion criteria were as follows: 1) no BMI data at diagnosis, 2) incomplete oncological data, 3) no regular follow-up, and 4) heterogenous carcinoma.

Table 1 Demographic and baseline characteristics of study cohort. (categorized by X-tile cutoffs)

\begin{tabular}{|c|c|c|c|c|c|}
\hline & Underweight $(n=216)$ & Normal $(n=968)$ & Overweight $(n=826)$ & total & $P$ value \\
\hline Age & $65(59-72)$ & $63(57-69)$ & $64(57-69)$ & $63(58-69)$ & 0.004 \\
\hline Male (\%) & $117(54.2)$ & $583(60.3)$ & $551(66.7)$ & $1251(62.2)$ & 0.001 \\
\hline ALB & $38(35-42)$ & $39(36-42)$ & $40(36-43)$ & $39(36-42)$ & 0.001 \\
\hline FBG & $5.68(5.00-6.79)$ & $6.04(5.35-7.41)$ & $6.10(5.43-7.55)$ & $6.03(5.32-7.40)$ & $<0.001$ \\
\hline CA199 & $165.3(37.1-766.9)$ & $152.2(39.0-484.6)$ & $163.8(41.7-563.7)$ & $161.1(40.2-552.2)$ & 0.34 \\
\hline $\mathrm{TB}$ & $16.9(11.4-75.8)$ & $17.0(11.3-76.7)$ & $16.6(11.8-70.3)$ & $16.8(11.6-72.6)$ & 0.935 \\
\hline Biliary drainage (\%) & $38(24.5)$ & $152(20.8)$ & $134(21.8)$ & $324(21.6)$ & 0.486 \\
\hline ASA Score (\%) & & & & & 0.394 \\
\hline 1 & $130(61.6)$ & $530(56.5)$ & $433(53.9)$ & $1093(56)$ & \\
\hline 2 & $65(30.8)$ & $334(35.6)$ & $297(37)$ & $696(35.7)$ & \\
\hline 3 & $14(6.6)$ & $63(6.7)$ & $67(8.4)$ & $144(7.4)$ & \\
\hline 4 & $2(0.9)$ & $11(1.2)$ & $6(0.7)$ & $19(1)$ & \\
\hline Tumor location (\%) & & & & & 0.495 \\
\hline Head & $129(59.7)$ & $538(55.6)$ & $473(57.2)$ & $1140(56.7)$ & \\
\hline Body/Tail & $87(40.3)$ & $430(44.4)$ & $353(42.8)$ & $870(43.3)$ & \\
\hline Diagnostic year (\%) & & & & & 0.758 \\
\hline 2013 & $9(4.2)$ & $35(3.6)$ & $32(3.9)$ & $76(3.8)$ & \\
\hline 2014 & $19(8.8)$ & $57(5.9)$ & $56(6.8)$ & $132(6.6)$ & \\
\hline 2015 & $22(10.2)$ & $105(10.8)$ & $80(9.7)$ & $207(10.3)$ & \\
\hline 2016 & $30(13.4)$ & $146(15.1)$ & $137(16.6)$ & $313(15.6)$ & \\
\hline 2017 & $48(22.7)$ & $199(20.6)$ & $149(18.0)$ & $396(19.7)$ & \\
\hline 2018 & $47(21.8)$ & $205(21.3)$ & $174(21.1)$ & $426(21.2)$ & \\
\hline 2019 & $41(19)$ & $221(22.8)$ & $198(24.0)$ & $460(22.9)$ & \\
\hline Stage & & & & & 0.162 \\
\hline la & $17(8.9)$ & $88(9.2)$ & $87(10.4)$ & $192(9.6)$ & \\
\hline $\mathrm{lb}$ & $48(22.2)$ & $222(22.9)$ & $208(25.2)$ & $478(23.8)$ & \\
\hline Ila & $19(8.8)$ & $72(7.4)$ & $64(7.8)$ & $155(7.7)$ & \\
\hline $11 \mathrm{~b}$ & $59(27.3)$ & $248(25.6)$ & $225(27.3)$ & $532(26.5)$ & \\
\hline III & $48(22.2)$ & $238(24.6)$ & $146(17.7)$ & $432(21.5)$ & \\
\hline IV & $25(11.3)$ & $100(10.3)$ & $96(11.6)$ & $221(11)$ & \\
\hline Differentiation (\%) & & & & & 0.252 \\
\hline I & $0(0)$ & $6(0.6)$ & $1(0.1)$ & $7(0.3)$ & \\
\hline$\|$ & $67(31)$ & $325(33.6)$ & $258(31.2)$ & $650(32.3)$ & \\
\hline III & $149(69)$ & $633(65.3)$ & $567(68.7)$ & $1349(67.1)$ & \\
\hline IV & $0(0)$ & $1(0.1)$ & $0(0)$ & $1(0)$ & \\
\hline Unknown & $0(0)$ & $3(0.3)$ & $0(0)$ & $3(0.1)$ & \\
\hline Chemotherapy (\%) & $93(43.1)$ & $57(56.9)$ & $500(60.5)$ & $1144(56.9)$ & $<0.001$ \\
\hline Surgical interventions (\%) & $152(70.4)$ & $669(69.1)$ & $598(72.4)$ & $1419(70.6)$ & 0.313 \\
\hline
\end{tabular}

ALB Albumin, FBG Fasten blood glucose, TB Total bilirubin 
Finally, 2010 patients were included in our study. (Supp. Figure 1).

The study protocol was approved by the institutional review board at the authors' affiliated hospital. The local ethics committee waived the need for informed consent because the study was observational and retrospective. The study was undertaken according to the Strengthening the Reporting of Observational Studies in Epidemiology (STROBE) guidelines [11] and in accordance with the latest version of the Declaration of Helsinki.

\section{Definition}

Height and weight were recorded at diagnosis. Body mass index was calculated as weight in kilograms divided by the square of height in meters. The cutoffs defined by X-tile were $18.9 \mathrm{~kg} / \mathrm{m}^{2}$ and $23.3 \mathrm{~kg} / \mathrm{m}^{2}$ for trinomial categorization. Additionally, BMI was categorized into three groups based on the cutoffs $\left(18.5 \mathrm{~kg} / \mathrm{m}^{2}\right.$ and $23 \mathrm{~kg} /$ $\mathrm{m}^{2}$ ) defined by the World Health Organization (WHO) for Asians [12]. Patients under $18.9 \mathrm{~kg} / \mathrm{m}^{2}$, between $18.9-23.3 \mathrm{~kg} / \mathrm{m}^{2}$ and over $23.3 \mathrm{~kg} / \mathrm{m}^{2}$ were respectively marked as underweight, normal and overweight. Similar categorization was performed based on WHO cutoffs. For better discrimination, different categorizing standards were marked using subscript text $\left(-_{\text {Xtile }}\right.$ or $\left.-_{\text {WHO }}\right)$. Disease was staged per the 8th American Joint Committee on Cancer (AJCC) guidelines using the TNM system. Treatment strategies were determined per NCCN guidelines. Surgeries were performed for patients with resectable tumors. For patients with locally advanced and borderline-resectable disease, treatment with neoadjuvant chemotherapy with or without subsequent surgical resection or upfront surgery was provided. For patients with metastatic disease, palliative and supportive care was provided. Biliary drainage included preoperative biliary stent placement, nasobilliary drainage and percutaneous transhepatic cholangial drainage. The chemotherapy mentioned in the RESULTS and DISCUSSION sections refers to the postoperative adjuvant chemotherapy strategy or the chemotherapy strategy for patients with unresectable or metastatic disease. The regimens of the postoperative chemotherapy strategy included gemcitabine only, gemcitabine + S-1, gemcitabine + capecitabine, gemcitabine+ oxaliplatin and gemcitabine+ nimotuzumab. The regimens used for patients with advanced disease were albumin paclitaxel+ gemcitabine and FOLFIRINOX. Overall survival (OS) was calculated in months and defined as the time from diagnosis to the date of death due to any cause.

\section{Statistical analysis}

Normally distributed continuous variables are presented as the mean $\pm \mathrm{SD}$ and were analyzed using Student's t-test. Nonnormally distributed continuous variables are presented as medians (quartile 1-quartile 3 (Q1-Q3)) and were analyzed using the Mann-Whitney $U$ test. The

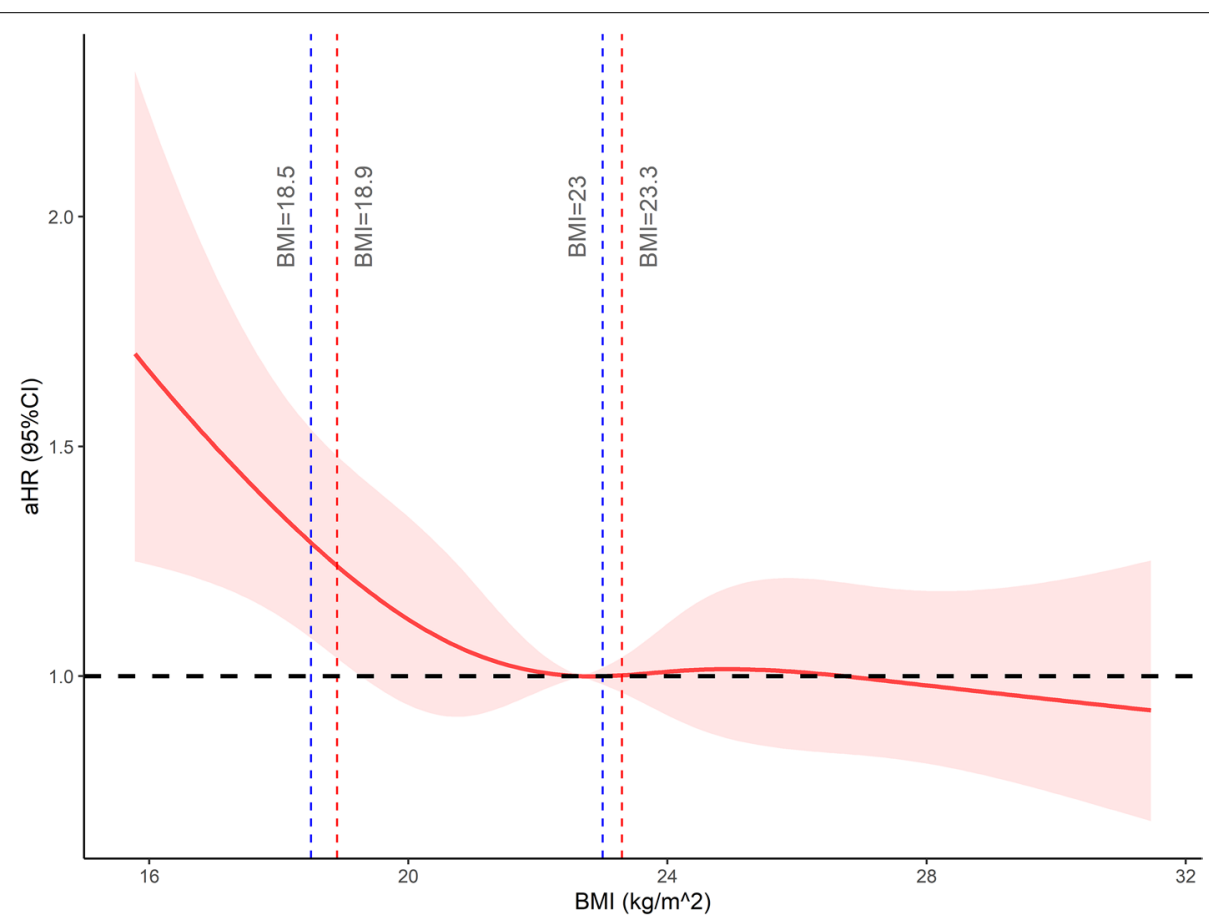

Fig. 1 The relationship between BMI and OS was explored using RCS function based on the Cox model over 5 knots. 95\% confidence bands are displayed 
Kolmogorov-Smirnov test was utilized for normality tests of continuous variables. Categorical variables are presented as percentages and were analyzed using Pearson's test. The proportional hazards assumption (PH assumption) was tested using the Schoenfeld partial residual method [13]. The OS was assessed using Kaplan-Meier curves and log-rank tests in the univariable analyses and restricted cubic spline (RCS) curves (continuous BMI variable) [14-16] and Cox proportional hazards models (continuous and categorical BMI variables) were used for multivariable analyses adjusted with the clinically relevant factors identified in the univariable analyses. The Cox-Mantel test was used for significance comparison. For better result presentation, in Cox regression models, CA199 was transformed into log (CA199). Moreover, due to the coherence of surgical intervention and TNM stages, surgical intervention was hidden in the multivariable Cox models and PS analyses. The knot number for RCS curves was set as 5 because of the sample size. X-tile was utilized to determine the cutoff value [17]. Associations (statistical "association" was marked as italic text) between categorical data were assessed using the $\chi^{2}$ test, associations between continuous data were assessed using the Spearman rank test, and associations between categorical data and continuous data were assessed using the Kruskal-Wallis test. For interaction (statistical "interaction" was marked as italic text) evaluation, the product term in the Cox model was utilized to assess the multiplicative interaction, while the relative excess risk due to interaction (RERI), the attributable proportion due to interaction (AP) and the synergy index (S) were used for the assessment of additive interaction $[18,19]$. If there is no biological interaction, RERI and AP are equal to 0 and $\mathrm{S}$ is equal to 1 .

To eliminate the imbalance of chemotherapy administration among BMI categories, inverse probability of treatment weighting (IPTW) and standardized mortality ratio weighting (SMRW) were also introduced for further analysis. The matching parameters included age, sex, ASA physical status, CA199, total bilirubin (TB), fasting blood glucose (FBG), albumin (ALB), biliary drainage, stage, differentiation and chemotherapy administration.

Statistical analyses were performed by SPSS (IBM SPSS Statistics 26.0), R Studio and X-Tile software. $P<0.050$ was regarded to be statistically significant.

\section{Results}

\section{Patients and characteristics}

2010 patients were enrolled in this study, and the baseline data were displayed in Table 1 and Supp. Table 1. The median BMI was $22.67 \mathrm{~kg} / \mathrm{m}^{2}$. The median OS time was 17.3 months, while the median follow-up duration was 33.3 months. Chemotherapy administered to $56.5 \%$ of the
Table 2 Univariate analysis of risks of OS

\begin{tabular}{|c|c|c|}
\hline & \multicolumn{2}{|l|}{ Univariate } \\
\hline & $\mathrm{HR}$ & $P$ value \\
\hline Age & $1.012(1.006-1.018)$ & $<0.001$ \\
\hline Male & $1.044(0.932-1.170)$ & 0.453 \\
\hline ALB & $0.991(0.981-1.002)$ & 0.106 \\
\hline FBG & $0.998(0.992-1.004)$ & 0.59 \\
\hline $\log C A 199$ & $1.318(1.236-1.406)$ & $<0.001$ \\
\hline $\mathrm{TB}$ & $1.000(1.000-1.001)$ & 0.164 \\
\hline Biliary drainage & $1.057(0.929-1.203)$ & 0.398 \\
\hline Body/tail & $1.085(0.971-1.212)$ & 0.149 \\
\hline Diagnostic year & & 0.103 \\
\hline 2013 & Ref. & \\
\hline 2014 & $1.029(0.762-1.389)$ & 0.852 \\
\hline 2015 & $1.204(0.910-1.594)$ & 0.194 \\
\hline 2016 & $0.937(0.715-1.228)$ & 0.637 \\
\hline 2017 & $1.120(0.858-1.460)$ & 0.405 \\
\hline 2018 & $1.153(0.880-1.512)$ & 0.301 \\
\hline 2019 & $0.960(0.707-1.304)$ & 0.795 \\
\hline ASA Score & & 0.798 \\
\hline 1 & Ref. & \\
\hline 2 & $0.977(0.867-1.102)$ & 0.709 \\
\hline 3 & $0.926(0.748-1.148)$ & 0.485 \\
\hline 4 & $1.180(0.707-1.968)$ & 0.527 \\
\hline Stage & & $<0.001$ \\
\hline la & Ref. & \\
\hline $\mathrm{lb}$ & $1.168(1.508-1.946)$ & 0.002 \\
\hline Ila & $1.618(1.186-2.207)$ & 0.002 \\
\hline$\| \mathrm{b}$ & $2.197(1.717-2.811)$ & $<0.001$ \\
\hline III & $3.213(2.512-4.110)$ & $<0.001$ \\
\hline IV & $6.307(4.831-8.235)$ & $<0.001$ \\
\hline Differentiation & & $<0.001$ \\
\hline 1 & Ref. & \\
\hline$\|$ & $2.645(0.372-18.835)$ & 0.331 \\
\hline III & $3.941(0.554-28.008)$ & 0.171 \\
\hline IV & $41.974(2.616-673.418)$ & 0.008 \\
\hline Unknown & $1.399(0.127-15.446)$ & 0.784 \\
\hline Chemotherapy & $0.511(0.458-0.570)$ & $<0.001$ \\
\hline Surgical intervention & $0.254(0.207-0.309)$ & $<0.001$ \\
\hline $\mathrm{BMl}$ & $0.964(0.946-0.983)$ & $<0.001$ \\
\hline X-Tile categorizing & & $<0.001$ \\
\hline$<18.9$ & Ref. & \\
\hline $18.9-23.3$ & $0.745(0.626-0.886)$ & 0.001 \\
\hline$\geq 23.3$ & $0.665(0.556-0.794)$ & $<0.001$ \\
\hline WHO categorizing & & $<0.001$ \\
\hline$<18.5$ & Ref. & \\
\hline $18.5-23$ & $0.625(0.514-0.759)$ & $<0.001$ \\
\hline$\geq 23$ & $0.563(0.462-0.685)$ & $<0.001$ \\
\hline
\end{tabular}

ALB Albumin, FBG Fasten blood glucose, TB Total bilirubin, HR Hazard ratio, Ref Reference 
patients. The whole cohort consisted of 192 Ia (9.6\%), 478 Ib (23.8\%), 155 IIa (7.7\%), 532 IIb (26.5\%), 432 III (21.5\%) and 221 IV (11\%) stage patients. For the X-tile categorization, the underweight, normal and overweight groups consisted of 216 (10.7\%), 968 (48.2\%) and 826 (41.1\%) patients, respectively. For the WHO categorization, these three categories included 157 (7.8\%), 943 (46.9\%) and 910 (45.3\%) patients, respectively. Significant differences were observed in age, sex, FBG and chemotherapy administration among the categories when analyzed with both strategies.

\section{Relationship of BMI with OS}

The association between BMI at diagnosis and the OS was depicted as RCS curves adjusted by the Cox model. (Fig. 1). The $P$ values of the total correlation and nonlinear correlation were 0.004 and 0.124 , respectively, suggesting that there existed a negatively correlated linear relationship between $\mathrm{BMI}$ and the adjusted hazard ratio (aHR). A sharp slope was observed at the beginning of the curve, and the curve tended to be smooth when BMI exceeded approximately $23 \mathrm{~kg} / \mathrm{m}^{2}$.

\section{BMI was a positive prognostic predictor in multivariable risk adjustment analysis}

As continuous data, BMI was tested fitting the $\mathrm{PH}$ assumption. (Pearson relevance: 0.036, $P=0.198$ ) (Supp. Figure 2) In the univariate Cox survival analysis, BMI was independently analyzed as continuous data, X-tile categorical data and WHO categorical data. BMI was significantly related with the OS in all analyses. (Table 2) (X-tile: hazard ratio (HR) (overweight vs. normal) $=0.892, P=0.058$; WHO: HR (overweight vs. normal) $=0.901, P=0.076$ )
Multivariable Cox regression models were established based on continuous, X-tile categorical and WHO categorical BMI data. (Fig. 2, Table 3) (X-tile: aHR (overweight vs. normal) $=0.880, P=0.045$; WHO: aHR (overweight vs. normal) $=0.903, P=0.102$ ) TNM stages, differentiation, CA199 and chemotherapy were significantly related to the OS in all Cox models. Both continuous and categorical BMI were discovered to be associated with chemotherapy (continuous BMI: association $P<0.001, \mathrm{X}$-tile categorical BMI: association $P<0.001$, WHO categorical BMI: association $P<0.001$ ). Moreover, the product term of categorical BMI and chemotherapy was significantly relevant to the OS (interaction $P_{X t i l e}=0.004 ;$ interaction $P_{W H O}=0.020$ ), suggesting the multiplicative interactions in the models. The additive interaction between BMI and chemotherapy was explored and confirmed (RERI $\mathrm{Xtile}_{\text {: }}$ : $0.50(0.30-0.70), \mathrm{AP}_{\text {Xtile }}: 0.58(0.29-0.86), \mathrm{S}_{\text {Xtile }}: 0.21$ (0.06-0.75); $\mathrm{RERI}_{\mathrm{WHO}}: 0.56(0.37-0.74), \mathrm{AP}_{\mathrm{WHO}}: 0.70$ (0.37-1.02), $\left.\mathrm{S}_{\mathrm{WHO}}: 0.26(0.12-0.57)\right)$. Nevertheless, neither association (Table 1, Supp. Table $1, P_{\text {Xtile }}=0.162$, $\left.P_{\mathrm{WHO}}=0.099\right) \quad$ nor interaction $\quad\left(P_{\mathrm{Xtile}}=0.699\right.$, $\left.P_{\mathrm{WHO}}=0.966\right)$ was observed between categorical BMI and TNM stage.

\section{Patients with higher BMI gain more OS benefits from chemotherapy}

In the different BMI categories, the ratio of patients receiving chemotherapy differed signifi-

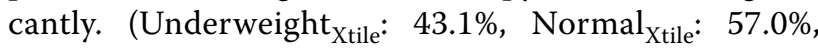
Overweight $_{\mathrm{Xtil}_{\mathrm{l}}}: \quad 60.5 \%, P<0.001$; Underweight ${ }_{\mathrm{WHO}}$ : $40.8 \%$, Normal ${ }_{\mathrm{WHO}}: 56.0 \%$, Overweight ${ }_{\mathrm{WHO}}: 60.7 \%$, $P<0.001)$. In different Cox models stratified by
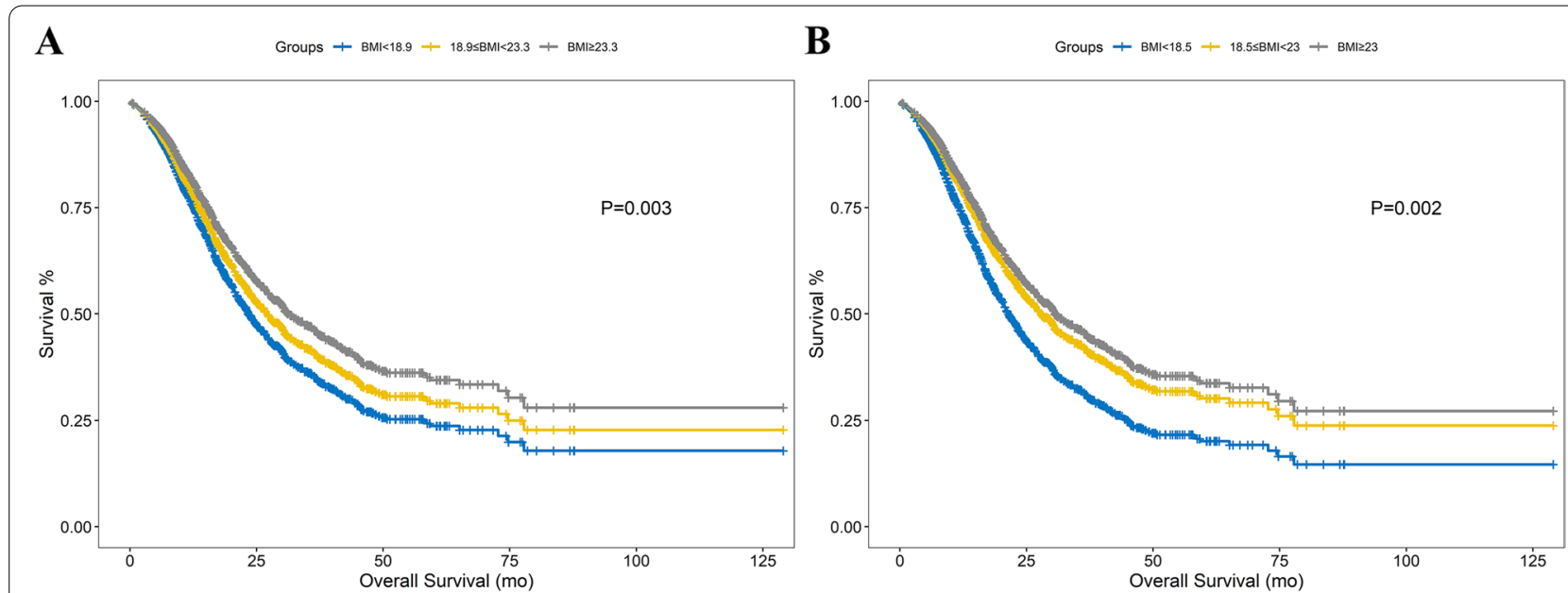

Fig. 2 A Adjusted survivorship curves of the Cox model with X-tile categorical BMI. B The adjusted survivorship curves of the Cox model with WHO categorical BMI 
Table 3 Multivariate analyses of risks of OS

\begin{tabular}{|c|c|c|c|c|c|c|}
\hline & \multicolumn{2}{|l|}{ Continuous BMI } & \multicolumn{2}{|l|}{ WHO Model } & \multicolumn{2}{|l|}{ X-tile Model } \\
\hline & aHR & $P$ value & aHR & $P$ value & aHR & $P$ value \\
\hline Age & $1.004(0.998-1.011)$ & 0.223 & $1.004(0.997-1.01)$ & 0.279 & $1.004(0.997-1.011)$ & 0.234 \\
\hline Male (female as Ref.) & $1.120(0.992-1.266)$ & 0.068 & $1.124(0.995-1.271)$ & 0.060 & $1.122(0.993-1.268)$ & 0.064 \\
\hline ALB & $1.004(0.992-1.017)$ & 0.51 & $1.004(0.992-1.016)$ & 0.541 & $1.005(0.993-1.017)$ & 0.43 \\
\hline FBG & $1.018(0.997-1.039)$ & 0.086 & $1.017(0.996-1.038)$ & 0.111 & $1.018(0.998-1.039)$ & 0.084 \\
\hline $\log C A 199$ & $1.164(1.093-1.240)$ & $<0.001$ & $1.161(1.09-1.236)$ & $<0.001$ & $1.162(1.091-1.237)$ & $<0.001$ \\
\hline TB & $1.000(0.999-1.001)$ & 0.986 & $1(0.999-1.001)$ & 0.902 & $1(0.999-1.001)$ & 0.899 \\
\hline Biliary drainage (no as Ref.) & $1.113(0.941-1.316)$ & 0.213 & $1.109(0.938-1.311)$ & 0.227 & $1.114(0.942-1.318)$ & 0.205 \\
\hline ASA Score & & 0.142 & & 0.163 & & 0.151 \\
\hline 1 & Ref. & & Ref. & & Ref. & \\
\hline 2 & $0.926(0.817-1.050)$ & 0.231 & $0.926(0.817-1.05)$ & 0.231 & $0.927(0.818-1.051)$ & 0.239 \\
\hline 3 & $1.182(0.934-1.495)$ & 0.164 & $1.179(0.931-1.493)$ & 0.171 & $1.184(0.935-1.498)$ & 0.16 \\
\hline 4 & $1.317(0.753-2.305)$ & 0.334 & $1.272(0.727-2.228)$ & 0.400 & $1.298(0.742-2.271)$ & 0.36 \\
\hline Stage & & $<0.001$ & & $<0.001$ & & $<0.001$ \\
\hline la & Ref. & & Ref. & & Ref. & \\
\hline $\mathrm{lb}$ & $1.587(1.210-2.080)$ & 0.001 & 1.58 (1.205-2.072) & 0.001 & $1.574(1.2-2.064)$ & 0.001 \\
\hline Ila & $1.704(1.226-2.370)$ & 0.002 & $1.681(1.209-2.337)$ & 0.002 & $1.691(1.216-2.351)$ & 0.002 \\
\hline$\| \mathrm{b}$ & $2.399(1.846-3.117)$ & $<0.001$ & $2.384(1.834-3.098)$ & $<0.001$ & 2.391 (1.84-3.107) & $<0.001$ \\
\hline III & $3.568(2.744-4.640)$ & $<0.001$ & $3.542(2.724-4.607)$ & $<0.001$ & $3.558(2.736-4.627)$ & $<0.001$ \\
\hline IV & $6.478(4.856-8.641)$ & $<0.001$ & $6.442(4.831-8.591)$ & $<0.001$ & $6.501(4.874-8.671)$ & $<0.001$ \\
\hline Differentiation & & $<0.001$ & & $<0.001$ & & $<0.001$ \\
\hline I & Ref. & & Ref. & & Ref. & \\
\hline$\|$ & $4.642(0.649-33.205)$ & 0.126 & $4.725(0.66-33.815)$ & 0.122 & $4.762(0.665-34.092)$ & 0.12 \\
\hline III & $6.444(0.903-46.008)$ & 0.063 & $6.562(0.918-46.882)$ & 0.061 & $6.637(0.929-47.436)$ & 0.059 \\
\hline IV & 1 & & 1 & & 1 & 1 \\
\hline Unknown & $1.652(0.147-18.599)$ & 0.684 & $1.744(0.155-19.613)$ & 0.652 & $1.744(0.155-19.61)$ & 0.652 \\
\hline Chemotherapy & $0.458(0.405-0.518)$ & $<0.001$ & $0.462(0.408-0.522)$ & $<0.001$ & $0.462(0.408-0.522)$ & $<0.001$ \\
\hline $\mathrm{BMI}$ & $0.972(0.953-0.991$ & 0.004 & & & & \\
\hline BMI Stage & & & & 0.002 & & 0.003 \\
\hline Underweight & & & Ref. & & Ref. & \\
\hline Normal & & & $0.759(0.617-0.935)$ & 0.010 & $0.821(0.682-0.989)$ & 0.038 \\
\hline Overweight & & & $0.685(0.555-0.847)$ & 0.000 & $0.723(0.597-0.875)$ & 0.001 \\
\hline
\end{tabular}

ALB Albumin, FBG Fasten blood glucose, TB Total bilirubin, aHR Adjusted hazard ratio, Ref Reference

categorical BMI, chemotherapy had different impacts on prognosis, indicating that the patients with higher BMI benefitted more from chemotherapy. (aHR: Underweight $_{\text {Xtile }}: 0.565 \quad(0.389-0.819), \quad$ Normal $_{X \text { tile }}$ : 0.474 (0.395-0.567), Overweight ${ }_{\text {Xtile: }} 0.409$ (0.3370.496); Underweight ${ }_{\mathrm{WHO}}: 0.613 \quad(0.401-0.940)$, Normal $_{\mathrm{WHO}}$ : $0.464 \quad(0.387-0.557)$, Overweight ${ }_{\mathrm{WHO}}$ : $0.425(0.353-0.512))$. Due to different chemotherapeutic strategies between patients with resectable and advanced disease, stratified by categorical BMI and resectability, we further confirmed the improved chemotherapy effect in patients with a higher BMI. (Supp. Table 2) Stratified by chemotherapy administration, it was discovered that categorical BMI was no longer associated with the OS in patients who did not receive chemotherapy in the multivariable Cox models. (Supp. Table 3, Supp. Table 4).

\section{Propensity score analysis revealed the role of BMI additionally}

IPTW and SMRW analyses were performed not only between the underweight category and normal category but also between the overweight category and normal category. The characteristics of the weighted cohorts were shown. (Table 4, Supp. Table 5, Supp. Table 6, Supp. Table 7) BMI was still an independent prognostic predictor with balanced chemotherapy status. (Fig. 3, Fig. 4). 
Table 4 Baseline data comparisons after IPTW analysis. (categorized by X-tile cutoffs)

\begin{tabular}{|c|c|c|c|c|c|c|c|c|}
\hline & $\begin{array}{l}\text { Underweight } \\
(1089.88)\end{array}$ & Normal (1091.45) & $P$ value & SMD & Normal (1672.01) & $\begin{array}{l}\text { Overweight } \\
(1668.85)\end{array}$ & $P$ value & SMD \\
\hline Age & $64(58-70)$ & $63(58-70)$ & 0.534 & 0.055 & $63.00(57.77-69.00)$ & $64.00(57.00-69.00)$ & 0.982 & 0.001 \\
\hline Female (\%) & $463.5(42.5)$ & $451.5(41.4)$ & 0.783 & 0.023 & $615.5(36.8)$ & $613.3(36.7)$ & 0.978 & 0.001 \\
\hline Asa (\%) & & & 1 & 0.01 & & & 1 & 0.003 \\
\hline 1 & $631.8(58.0)$ & $635.6(58.2)$ & & & $934.9(55.9)$ & $934.5(56.0)$ & & \\
\hline 2 & $383.3(35.2)$ & $379.3(34.8)$ & & & $599.6(35.9)$ & $597.6(35.8)$ & & \\
\hline 3 & $65.0(6.0)$ & $66.5(6.1)$ & & & $123.3(7.4)$ & $122.3(7.3)$ & & \\
\hline 4 & $9.8(0.9)$ & $10.0(0.9)$ & & & $14.3(0.9)$ & $14.5(0.9)$ & & \\
\hline Differentiation (\%) & & & 0.266 & 0.109 & & & 0.985 & 0.009 \\
\hline । & $0.0(0.0)$ & $6.0(0.5)$ & & & $7.0(0.4)$ & $6.1(0.4)$ & & \\
\hline$\|$ & $345.2(31.7)$ & $358.7(32.9)$ & & & $534.5(32.0)$ & $531.6(31.9)$ & & \\
\hline III & $744.7(68.3)$ & $726.7(66.6)$ & & & $1130.5(67.6)$ & $1131.2(67.8)$ & & \\
\hline Stage (\%) & & & 0.989 & 0.064 & & & 1 & 0.003 \\
\hline la & $97.7(9.0)$ & $98.4(9.0)$ & & & $162.2(9.7)$ & $162.6(9.7)$ & & \\
\hline $\mathrm{lb}$ & $237.0(21.7)$ & $247.4(22.7)$ & & & $401.2(24.0)$ & $401.3(24.0)$ & & \\
\hline Ila & $86.1(7.9)$ & $85.2(7.8)$ & & & $128.6(7.7)$ & $128.6(7.7)$ & & \\
\hline$\| \mathrm{b}$ & $256.9(23.6)$ & $272.3(24.9)$ & & & $431.5(25.8)$ & $431.3(25.8)$ & & \\
\hline III & $269.5(24.7)$ & $265.5(24.3)$ & & & $359.5(21.5)$ & $357.6(21.4)$ & & \\
\hline IV & $142.7(13.1)$ & $122.6(11.2)$ & & & $188.9(11.3)$ & $187.5(11.2)$ & & \\
\hline Biliary drainage (\%) & $188.1(17.3)$ & $179.0(16.4)$ & 0.785 & 0.023 & $275.4(16.5)$ & $274.0(16.4)$ & 0.978 & 0.001 \\
\hline TB & $17.10(11.36-65.50)$ & $16.60(11.20-66.31)$ & 0.675 & 0.018 & $16.70(11.22-65.63)$ & $16.33(11.70-66.45)$ & 0.989 & 0.001 \\
\hline AIB & $39.00(36.00-42.00)$ & $39.00(36.00-42.00)$ & 0.711 & 0.011 & $39.00(37.00-42.00)$ & $39.00(36.00-43.00)$ & 0.958 & 0.003 \\
\hline FBG & $5.82(5.08-7.26)$ & $5.99(5.30-7.35)$ & 0.489 & 0.065 & $6.06(5.36-7.43)$ & $6.14(5.45-7.55)$ & 1 & $<0.001$ \\
\hline chemotherapy (\%) & $587.3(53.9)$ & $598.8(54.9)$ & 0.816 & 0.02 & $989.8(59.2)$ & $990.0(59.3)$ & 0.959 & 0.003 \\
\hline CA199 & $167.53(41.57-736.56)$ & $152.50(39.30-481.59)$ & 0.225 & 0.016 & $149.90(39.30-465.31)$ & $164.78(41.49-561.24)$ & 0.925 & 0.005 \\
\hline
\end{tabular}

ALB Albumin, FBG Fasten blood glucose, TB Total bilirubin, SMD Standard deviation mean difference

\section{Discussion}

BMI, a simple, inexpensive and readily available parameter, has been broadly applied in the evaluation of diseases, such as metabolic, endocrine, and oncological diseases [20-24]. The role of BMI in pancreatic cancer oncogenesis has been previously studied and discussed. Higher BMI is related to an increased incidence of pancreatic cancer [3, 4]. But for patients diagnosed with pancreatic cancer, how BMI influences their prognoses is still unclear and controversial.

Okura T. et al. and Tsai S. et al. reported the positive impact of high BMI on PDAC patients' prognoses [9, 25]. However, the sample size of Dr. Okura T.s work was limited. In Dr. Tsai's work, researchers analyzed the OS and BMI using Cox models without chemotherapy parameter included. Li D., Yuan C. and McWilliams R.R. held different opinions [26-28]. In Dr. McWilliams' research, the study cohort was characterized by the advanced stage and high average BMI (stage III\&IV: 69.9\%, BMI: 27.6 (24.4-31.2) $\left.\mathrm{kg} / \mathrm{m}^{2}, \mathrm{BMI}<18.5: 0.7 \%\right)$. In addition, chemotherapy administration was not discussed or analyzed. In the studies of Dr. Li and Dr. Yuan, the early-onset pancreatic cancer pattern [29], the lack of adjuvant/neoadjuvant treatment data and the baseline data differences might explain the differences between their results and ours. In some studies, it was proposed that no association existed between BMI and the OS [10, 30-32]. Sample size, covariates included in the studies and racial/ regional differences might lead to conclusion divergence. Above all, the differences between previous works and ours could be explained by baseline data differences (e.g., racial/regional background, TNM stages), the covariates included and the sample size. In addition, the advancement of chemotherapy is another possible cause for the conclusion divergence due to the vital role of chemotherapy in the protective impact of BMI on the OS .

In the RCS analysis, a negative linear relationship between BMI and aHR was observed. There was a strong association with a higher risk of death in underweight patients than in normal weight individuals. The curve tends to be smooth when BMI is above $\sim 23 \mathrm{~kg} / \mathrm{m}^{2}$. In the univariate analysis, the tumor location and the diagnostic year were filtered out for the multivariable analysis. Categorical BMI grouped by both standards and continuous 


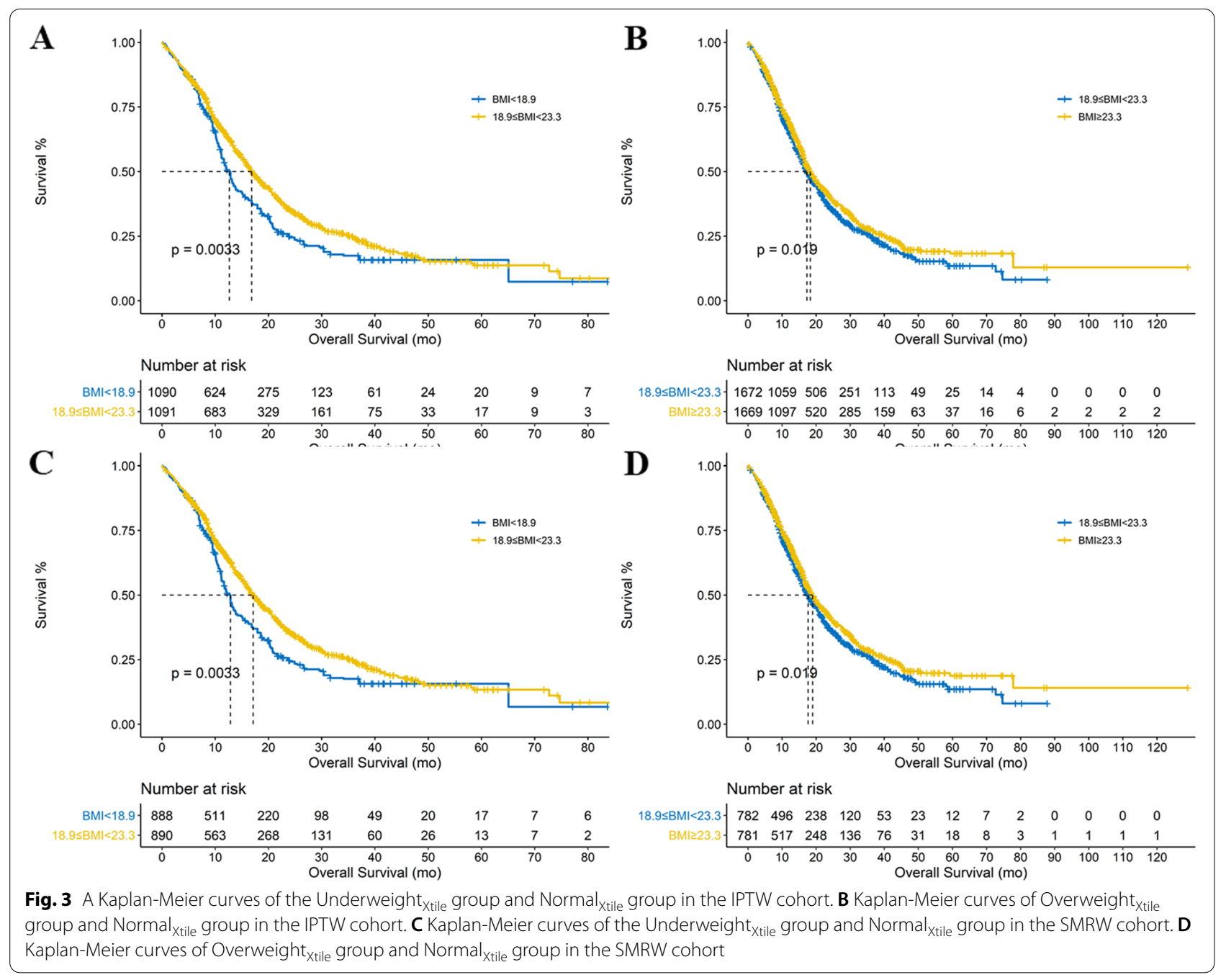

BMI were significantly associated with the OS. FBG and ALB were 2 additional parameters used to evaluate the nutrition condition. Neither the significant impact on the OS (Table 3) nor the correlation with BMI (FBG: $P=0.087$, ALB: $P=0.060$ ) was observed in this study.

Chemotherapy was discovered to be related to both continuous BMI and categorical BMI. No significant association or interaction was observed between TNM stage and BMI. More patients were treated with chemotherapy in the higher BMI groups. Stratified by BMI level, we compared the impact of chemotherapy in different categories. Chemotherapy had a more positive impact on the OS in the higher BMI categories. After further stratification by different chemotherapy strategies, a similar phenomenon was still observed. Stratified by chemotherapy administrated or not, the protective impact of BMI was only observed in the chemotherapy-administered subcategory. Above all, we discovered that higher BMI might prolong the OS time on the basis of chemotherapy.
And we hypothesized that this protective effect was probably via the improved response to chemotherapy.

Given that there existed an interaction and association between BMI and chemotherapy, IPTW analysis was then introduced as a sensitivity analysis. With pairwise comparisons, compared with normal-weight patients, being underweight was discovered to be a risk factor, while being overweight was a protective factor. Provided that the chemotherapy acceptance was matched in different categories, the results furtherly support our hypothesis that higher BMI conferred patients better responses to chemotherapy. The consistency of the IPTW and SMRW analysis results indicated that no significant confounding/interacting factor was excluded.

Some limitations in this study should be stressed. First, though PS analysis and multivariable risk adjustment were performed, the retrospective single-center data still limited the reliability and extrapolation of this study. A multicenter prospective study analyzing BMI could help 


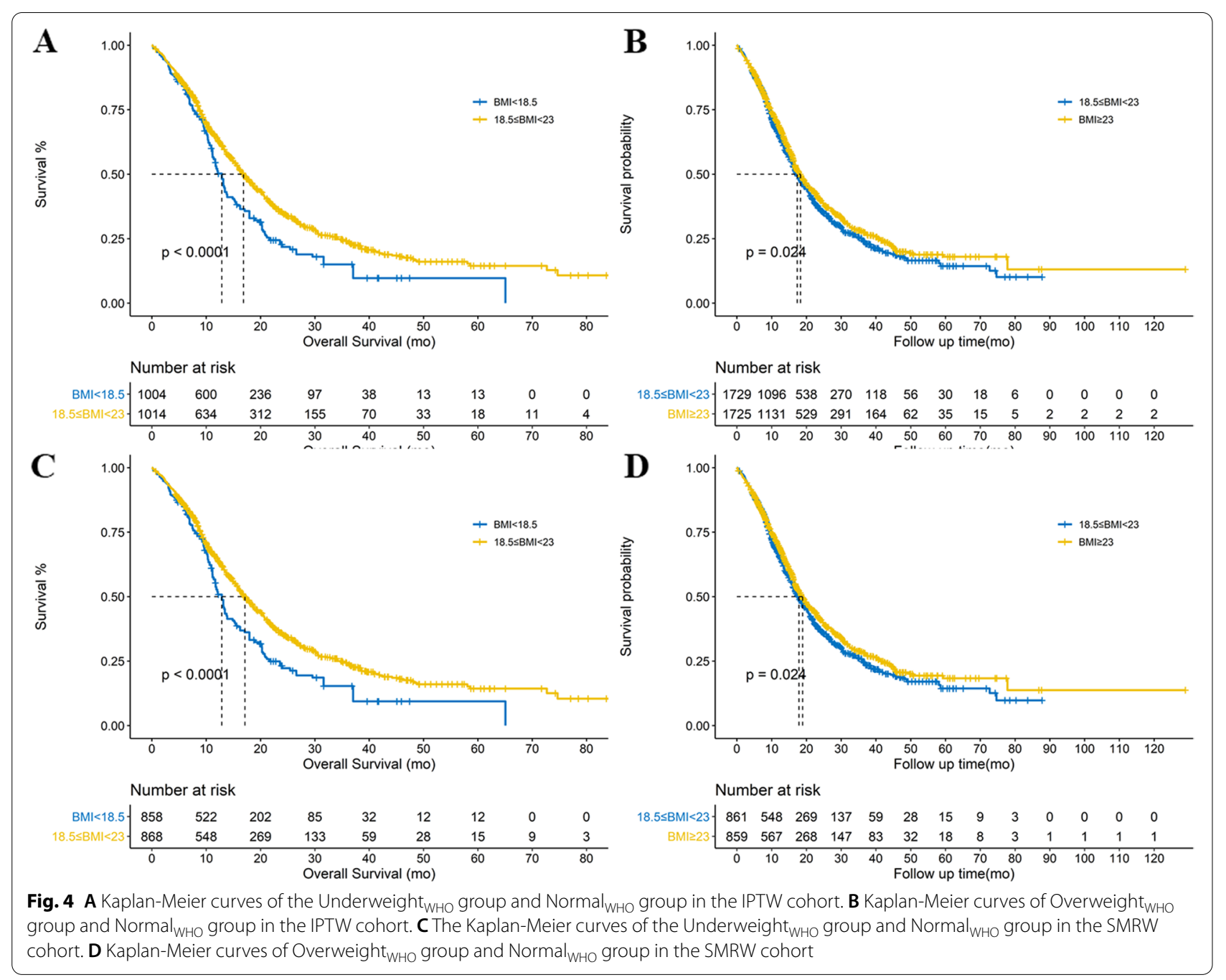

validate the results. Second, given that all enrolled subjects were from the Chinese Han population, further research on patients from different districts and races should be conducted. Additionally, the duration and courses of chemotherapy were not listed and analyzed. Sufficient or insufficient chemotherapy courses might lead to different outcomes, and how BMI influences these outcomes was undefined. Finally, selection bias may be present due to the preference for patients with resectable disease at our pancreatic disease center. However, with multivariate, PS and stratified analyses, we sought to fix the bias for reliable conclusions.

In this study, we elaborated on the relationship between BMI and the OS of PDAC patients based on IPTW, RCS and multivariable risk adjustment analyses and shed light on the probable underlying mechanism. Higher BMI indicated more frequent chemotherapy treatment and longer OS among PDAC patients. Among the different BMI categories, patients with a higher BMI also benefitted more from chemotherapy. The protective impact of high BMI disappeared when chemotherapy was not applied. Thus we hypothesized that high BMI might affect the OS of PDAC patients via the acceptance and response of chemotherapy. The relevance between $\mathrm{BMI}$ and chemotherapy requires further study.

\section{Supplementary Information}

The online version contains supplementary material available at https://doi. org/10.1186/s12885-021-09056-0.

Additional file 1: Supplementary Fig. 1. The flow-chart of case enrollment.

Additional file 2: Supplementary Table 1. Demographic and baseline characteristics of study cohort. (Categorized by WHO cutoffs).

Additional file 3: Supplementary Fig. 2. The Schoenfeld residual plot of $\mathrm{BMI}$ with rank of OS for $\mathrm{PH}$ assumption test.

Additional file 4: Supp. Table 2. Chemotherapy impact on OS stratified with categorical BMI and resectability. 
Additional file 5: Supp. Table 3. Multivariate analyses of risks of OS stratified with chemotherapy administration (categorized by Xtile cutoffs).

Additional file 6: Supp. Table 4. Multivariate analyses of risks of OS stratified with chemotherapy administration (categorized by WHO cutoffs).

Additional file 7: Supplementary Table 5. Baseline data comparisons after SMRW analysis. (categorized by Xtile cutoffs).

Additional file 8: Supplementary Table 6. Baseline data comparisons after IPTW analysis. (categorized by WHO cutoffs).

Additional file 9: Supplementary Table 7. Baseline data comparisons after SMRW analysis. (categorized by WHO cutoffs).

\section{Acknowledgments}

All included patients are gratefully acknowledged. Prof. Chenghong Peng, Dr. Zhiwei Xu and Dr. Jiabing Jin should be additionally acknowledged for their contributions in patients' management and data collection. Also, we appreciated Dr. Yusheng Shi so much for his suggestions in study design. Finally, we are respectful and thankful to all inclusive patients for their supports and devotions on the medicine.

\section{Authors' contributions}

Study concepts Ningzhen FU, Hao CHEN; Study design Ningzhen FU, Yu JIANG, Baiyong SHEN; Data acquisition Ningzhen FU, Kai QIN; Quality control of data and algorithms Ningzhen FU, Xiaxing DENG; Data analysis and interpretation Ningzhen FU, Xiaxing DENG; Statistical analysis Ningzhen FU, Yu JIANG; Manuscript preparation Ningzhen FU, Hao CHEN; Manuscript editing Ningzhen FU, Baiyong SHEN; Manuscript review Hao CHEN, Baiyong SHEN. The author(s) read and approved the final manuscript.

\section{Funding}

This study was supported in part by the National Natural Science Foundation of China (Grant number: 81871906).

\section{Availability of data and materials}

The raw data should be inaccessible to the public. The statistical data and results are available. Data can be requested by contacting fnz01 b74@rijh.com. cn.

\section{Declarations}

\section{Ethics approval and consent to participate}

All clinical decisions or interventions were in accordance with National Comprehensive Cancer Network (NCCN) guidelines for pancreatic cancer. The ethics committee of Shanghai Jiao Tong University School of Medicine affiliated with Ruijin Hospital waived the need for informed consent because the study was observational and retrospective. The ethics committee of Shanghai Jiao Tong University School of Medicine Affiliated Ruijin Hospital approved this retrospective study, including patients from the Pancreatic Disease Center.

\section{Consent for publication}

All coauthors consent to publication.

\section{Competing interests}

All coauthors declare no conflicts of interest.

\section{Author details}

'Department of General Surgery, Pancreatic Disease Center, Shanghai Ruijin Hospital affiliated with Shanghai Jiao Tong University School of Medicine, No.197 Ruijin Er Road, Shanghai 200025, China. ${ }^{2}$ Research Institute of Pancreatic Diseases, Shanghai Jiao Tong University School of Medicine, No.197 Ruijin Er Road, Shanghai 200025, China. ${ }^{3}$ State Key Laboratory of Oncogenes and Related Genes, No.197 Ruijin Er Road, Shanghai 200025, China. ${ }^{4}$ Institute of Translational Medicine, Shanghai Jiao Tong University, Shanghai, China.

Received: 25 February 2021 Accepted: 18 November 2021

Published online: 09 December 2021

\section{References}

1. Siegel RL, Miller KD, Jemal A. Cancer statistics, 2020. CA Cancer J Clin. 2020;70(1):7-30.

2. Luo G, Fan Z, Gong Y, et al. Characteristics and outcomes of pancreatic cancer by histological subtypes. Pancreas. 2019;48(6):817-22.

3. Carreras-Torres R, Johansson M, Gaborieau V, et al. The Role of Obesity, Type 2 diabetes, and metabolic factors in pancreatic cancer: a mendelian randomization study. J Natl Cancer Inst. 2017;109(9):djx012.

4. Untawale $\mathrm{S}$, Odegaard AO, Koh WP, et al. Body mass index and risk of pancreatic cancer in a Chinese population. Plos One. 2014;9(1):e85149.

5. Weng $Y$, Jiang $Y$, Fu N, et al. Oncological outcomes of robotic-assisted versus open pancreatoduodenectomy for pancreatic ductal adenocarcinoma: a propensity score-matched analysis. Surg Endosc. 2021;35(7).

6. Han J, Zhou Y, Zheng Y, et al. Positive effect of higher adult body mass index on overall survival of digestive system cancers except pancreatic cancer: a systematic review and meta-analysis. Biomed Res Int. 2017;2017:1049602.

7. Teoule P, Rasbach E, Oweira H, et al. Obesity and Pancreatic Cancer: A Matched-Pair Survival Analysis. J Clin Med. 2020;9(11):3526.

8. Shi YQ, Yang J, Du P, et al. Effect of body mass index on overall survival of pancreatic Cancer: a Meta-analysis. Medicine (Baltimore). 2016;95(14):e3305.

9. Okura T, Fujii M, Shiode J, et al. Impact of body mass index on survival of pancreatic cancer patients in Japan. Acta Med Okayama. 2018;72(2):129-35

10. Jiang QL, Wang CF, Tian YT, et al. Body mass index does not affect the survival of pancreatic cancer patients. World J Gastroenterol. 2017;23(34):6287-93.

11. von Elm E, Altman DG, Egger M, et al. The strengthening the reporting of observational studies in epidemiology (STROBE) statement: guidelines for reporting observational studies. Int J Surg. 2014;12(12):1495-9.

12. Consultation WE. Appropriate body-mass index for Asian populations and its implications for policy and intervention strategies. Lancet. 2004;363(9403):157-63.

13. Zhang Z, Reinikainen J, Adeleke KA, et al. Time-varying covariates and coefficients in cox regression models. Ann Transl Med. 2018;6(7):121.

14. Gurrin LC, Scurrah KJ, Hazelton ML. Tutorial in biostatistics: spline smoothing with linear mixed models. Stat Med. 2005;24(21):3361-81.

15. Desquilbet $L$, Mariotti $F$. Dose-response analyses using restricted cubic spline functions in public health research. Stat Med. 2010;29(9):1037-57.

16. Roshani D, Ghaderi E. Comparing smoothing techniques for fitting the nonlinear effect of covariate in cox models. Acta Inform Med. 2016;24(1):38-41.

17. Camp RL, Dolled-Filhart M, Rimm DL. X-tile: a new bio-informatics tool for biomarker assessment and outcome-based cut-point optimization. Clin Cancer Res. 2004;10(21):7252-9.

18. Kallberg H, Ahlbom A, Alfredsson L. Calculating measures of biological interaction using R. Eur J Epidemiol. 2006;21(8):571-3.

19. Andersson T, Alfredsson $L$, Kallberg $H$, et al. Calculating measures of biological interaction. Eur J Epidemiol. 2005;20(7):575-9.

20. Jiang W, Wang J, Shen $X$, et al. Establishment and validation of a risk prediction model for early diabetic kidney disease based on a systematic review and meta-analysis of 20 cohorts. Diabetes Care. 2020;43(4):925-33.

21. Corder K, Winpenny EM, Foubister C, et al. Becoming a parent: a systematic review and meta-analysis of changes in BMI, diet, and physical activity. Obes Rev. 2020;21(4):e12959.

22. Ford ME, Bauza CE, Findlay VJ, et al. BMI, physical activity, and breast cancer subtype in white, black, and Sea Island breast cancer survivors. Adv Cancer Res. 2020;146:83-102.

23. Floris G, Richard F, Hamy AS, et al. Body mass index and tumor-infiltrating lymphocytes in triple-negative breast Cancer. J Natl Cancer Inst. 2020.

24. Shepshelovich D, Xu W, Lu L, et al. Body mass index (BMI), BMI change, and overall survival in patients with SCLC and NSCLC: a pooled analysis of the international lung Cancer consortium. J Thorac Oncol. 2019;14(9):1594-607.

25. Tsai S, Choti MA, Assumpcao L, et al. Impact of obesity on perioperative outcomes and survival following pancreaticoduodenectomy for pancreatic cancer: a large single-institution study. J Gastrointest Surg. 2010;14(7):1143-50. 
26. Li D, Morris JS, Liu J, et al. Body mass index and risk, age of onset, and survival in patients with pancreatic cancer. JAMA. 2009;301(24):2553-62.

27. McWilliams RR, Matsumoto ME, Burch PA, et al. Obesity adversely affects survival in pancreatic cancer patients. Cancer. 2010;116(21):5054-62.

28. Yuan $C, B a o$ Y, Wu C, et al. Prediagnostic body mass index and pancreatic cancer survival. J Clin Oncol. 2013;31(33):4229-34.

29. Varghese AM, Singh I, Singh R, et al. Early-onset pancreas Cancer: clinical descriptors, genomics, and outcomes. J Natl Cancer Inst. 2021.

30. Gong Z, Holly EA, Bracci PM. Obesity and survival in population-based patients with pancreatic cancer in the San Francisco Bay Area. Cancer Causes Control. 2012;23(12):1929-37.

31. Olson SH, Chou JF, Ludwig E, et al. Allergies, obesity, other risk factors and survival from pancreatic cancer. Int J Cancer. 2010;127(10):2412-9.

32. Kasenda B, Bass A, Koeberle D, et al. Survival in overweight patients with advanced pancreatic carcinoma: a multicentre cohort study. BMC Cancer. 2014;14:728.

\section{Publisher's Note}

Springer Nature remains neutral with regard to jurisdictional claims in published maps and institutional affiliations.

- fast, convenient online submission

- thorough peer review by experienced researchers in your field

- rapid publication on acceptance

- support for research data, including large and complex data types

- gold Open Access which fosters wider collaboration and increased citations

- maximum visibility for your research: over 100M website views per year

At BMC, research is always in progress.

Learn more biomedcentral.com/submissions 